\title{
Medo de cair em idosos classificados como vulneráveis de um centro de referência à atenção da saúde do idoso
}

\section{Fear of falling among elderly people classified as vulnerable in a referral center for elderly health care}

(D) Miguel Jânio Costa Ferreira ${ }^{1}$, (D) Júlio César Queiroz de França ${ }^{1}$, (D)Juan Carlos Costa Mata Lobos ${ }^{1}$, (D) Roseanne Maria Silva Barbosa Santana ${ }^{1}$, (D) Juarez Belmiro Moraes Júnior ${ }^{1}$, (D)Andréa Santana Carneiro ${ }^{1}$, (DSilvio Gomes Monteiro ${ }^{1}$, (D) Maria Claudia Gonçalves $^{1}$

1 Universidade Ceuma - UNICEUMA

Correspondência

Miguel Jânio Costa Ferreira

E-mail: mfpersonal2010@hotmail.com

Submetido: 14 Junho 2020

Aceito: 14 Agosto 2020

Como citar

Ferreira MJC, França JCQ, Lobos JCCM, Santana RMSB, Moraes Júnior JB, Carneiro AS, et al. Medo de cair em idosos classificados como vulneráveis de um centro de referência à atenção da saúde do idoso. Acta Fisiatr. 2020;27(2):82-88.

DOI: 10.11606/issn.2317-0190.v27i2a171038

\section{RESUMO}

Objetivo: Correlacionar o medo de cair com a vulnerabilidade em idosos. Método: A vulnerabilidade foi avaliada por meio da Pesquisa sobre Idosos Vulneráveis (VES-13) e o medo de cair pela Escala de Eficácia de Quedas - Internacional - Brasileira (FES-I-BR), participaram idosos de ambos os sexos e idade (60-85 anos), foram excluídos voluntários com limitações cognitivas e os que não participavam do grupo de convivência. Foram aplicado os testes de Kolmogorov-Smirnov, de Mann-Whitney, de Spearman, de regressão logística binária e para análise de sensibilidade e especificidade da FES-I-BR, empregou-se a curva da característica do operador receptor (ROC), a análise foi realizada em $95 \%$ nível de confiança e $p<0,05$ foi adotado. Resultados: Entre os $n=123$ idosos, foi observado que $(33,3 \%)$ são vulneráveis, $n=120 \quad(97,6 \%)$ apresentaram alguma preocupação em cair. Entre as atividades analisadas na FES-I-BR, 21,1\% estão extremamente preocupados em cair ao andar em superfícies escorregadias e foi observada uma correlação positiva e significativa entre a pontuação do VES-13 com a pontuação da FES-I-BR, a FES-I-BR foi um previsor significativo para a vulnerabilidade. No presente estudo, a FES-I-BR de forma aceitável mostrou-se capaz de discriminar o medo de cair de vulneráveis de não vulneráveis em uma área de 0,656 ( $<<0,005)$, sensibilidade de $53,7 \%$ e especificidade de $69,5 \%$. Conclusão: 0 medo de cair está correlacionado com a vulnerabilidade, os idosos vulneráveis apresentaram mais medo de cair, o medo de cair é fator preditor de vulnerabilidade e a FES-I-BR foi capaz de discriminar o medo de cair de idosos vulneráveis de não vulneráveis.

Palavras chaves: Idoso, Serviços de Saúde para Idosos, Acidentes por Quedas, Medo

\begin{abstract}
Objective: Was to correlate the fear of falling with vulnerability in the elderly. Method: Vulnerability was assessed using the Vulnerable Elderly Survey (VES-13) and the fear of falling using the Falls Efficacy Scale - International - Brazilian (FES-I-BR), elderly people of both sexes and age (60- 85 years old), volunteers with cognitive limitations and those who did not participate in the coexistence group were excluded. The Kolmogorov-Smirnov, Mann-Whitney, Spearman, binary logistic regression tests were applied and for the sensitivity and specificity analysis of the FES-I-BR, the Receiver Operator Characteristic Curve (ROC) was used, analysis was performed at 95\% confidence level and $p<0.05$ was adopted. Results: Among the $n=123$ elderly, it was observed that (33.3\%) are vulnerable, $\mathrm{n}=120(97.6 \%)$ had some concern about falling. Among the activities analyzed at FES-I$B R, 21.1 \%$ are extremely concerned about falling when walking on slippery surfaces and a positive and significant correlation was observed between the VES-13 score and the FESI-BR score, FES-I-BR was a significant predictor for the vulnerability. In the present study, the FES-I-BR in an acceptable way proved to be able to discriminate the fear of falling from vulnerable to non-vulnerable in an area of 0.656 ( $p<0.005)$, sensitivity of $53.7 \%$ and specificity of $69,5 \%$. Conclusion: Fear of falling is correlated with vulnerability, vulnerable elderly people were more afraid of falling, fear of falling is a predictor of vulnerability and FES-I-BR was able to discriminate between the fear of falling of vulnerable and nonvulnerable elderly people.
\end{abstract}

Key words: Aged, Health Services for the Aged, Accidental Falls, Fear 


\section{INTRODUÇÃO}

Na transição demográfica, a população com 60 anos ou mais aumenta em proporções mais acelerada do que as outras faixas etárias. Este fenômeno acontece tanto em países desenvolvidos quanto em países em desenvolvimento como o Brasil. ${ }^{1}$

$\mathrm{Na}$ atualidade, o Brasil já alcançou os $12,5 \%$ da população em 2015 e uma probabilidade de aumento para 30\% até 2050, ${ }^{2}$ essa dinâmica posicionará o Brasil como a sexta população de idosos no planeta. ${ }^{3}$

Diante deste fenômeno populacional o interesse pela comunidade cientifica aumentou e as investigações para entender $o$ processo de envelhecimento provocaram mudanças nos processos avaliativos. ${ }^{4,5}$ As avaliações geriátricas, tem como propósito identificar os riscos relacionados a qualidade de vida e a saúde desta população. ${ }^{6}$

$O$ reconhecimento da vulnerabilidade, do risco de quedas e suas manifestações negativas no idoso tem sido amplamente investigado. ${ }^{6}$ Neste sentido, identificar a vulnerabilidade no idoso facilita as medidas protetora diante dos fatores de riscos relacionados a quedas e na intervenção profissional. ${ }^{1}$

Uma das características do idoso vulnerável está relacionado ao medo de cair, ${ }^{7}$ embora este sentimento seja desenvolvido por idosos que experimentaram ou não algum episodio, poucas informações estão descrita na literatura e são vários os questionamentos desenvolvidos sobre a vulnerabilidade e o medo de cair. ${ }^{1,8}$

O medo de cair é caracterizado na literatura como um sentimento preditor real a um evento imaginário ou aparente a quedas. ${ }^{9}$ As quedas em idosos retratam um dos grandes problemas na saúde pública, com números aproximados de $30 \%$ das pessoas com idades de 65 anos ou superior caírem pelo menos uma vez a cada ano, tendo uma frequência superior no sexo feminino e a incidência acentua-se com o avanço da idade desencadeando muitas complicações. ${ }^{6}$

Quedas são responsáveis pela quinta causa de morte provocados pelas Lesões não intencionais em indivíduos com idade igual ou superior a 65 anos, representa aproximadamente $40 \%$ das recorrências hospitalares e quase $50 \%$ das institucionalizações depois da alta hospitalar. ${ }^{10,11}$

A queda é definida como um deslocamento não intencional para uma posição inferior em relação a posição inicial, sendo provocada de forma multifatorial. ${ }^{11} \mathrm{Na}$ literatura os fatores intrínsecos ou extrínsecos associados ou não são as causas dos acidentes. $^{12}$

A Organização Mundial de Saúde (OMS) descreve como fatores de riscos para quedas os fatores biológicos (idade, sexo, doenças crónicas, declínio nas capacidades física, cognitiva e afetiva); comportamentais (polimedicação, falta de atividade física, calçado inadequado e alcoolismo); ambientais (edificações não planejas ou adaptadas) e socioeconómicos (baixa renda; condições habitacionais insatisfatórias pra vida; isolamento social, acesso limitado aos serviços de saúde e sociais). ${ }^{12}$

Diante do exposto, hipotetizamos que idosos vulneráveis estão mais preocupados em cair que idosos não vulneráveis.

\section{OBJETIVO}

O objetivo da pesquisa é correlacionar o medo de cair com a vulnerabilidade de idosos, além de verificar se existe correlações entre a classificação do VES-13 com os dados sociodemográficos.

\section{MÉTODOS}

Trata-se de um estudo de corte transversal descritivo e analítico, realizado no ano de 2019 em um centro de referência à atenção integral à saúde do idoso em São Luís, Maranhão, onde os idosos participam de atividades físicas, cognitivas e laborais entre três à cinco vezes por semana com duração mínima de quarenta minutos por atividade.

Fizeram parte desta pesquisa idosos que frequentassem o centro de referência integral ao idoso pelo menos três vezes por semana, foram incluídos aqueles que estivessem na etária entre 60-85 anos, de ambos os sexos, fizessem as atividades propostas pelo menos três vezes por semana, com ou sem histórico de quedas no último ano.

Foram excluídos aqueles que não participassem das atividades físicas desenvolvidas pelo grupo de convivência e portadores de limitações cognitivas.

Este estudo atendeu aos requisitos propostos pela Resolução no 466, de 12 de dezembro de 2012, do Conselho Nacional de Saúde (CNS) e foi apresentada ao comitê de ética da Universidade Ceuma e teve sua iniciação após a aprovação através do parecer consubstanciado $\mathrm{n}$ 0 2.851.570/2018.

Para a coleta de dados sociodemográficos, o procedimento seguiu uma dinâmica em formato em entrevista por meio de formulários desenvolvido seguindo as diretrizes de preenchimento da caderneta do idoso brasileira ${ }^{13}$ estruturado com variáveis como a idade, sexo, raça/cor, nível de escolaridade, situação conjugal, problemas de saúde, histórico de quedas nos últimos 12 meses e presença de limitações cognitivas sinalizadas em condições frequentes "limitações cognitivas (demência)" na caderneta de cada voluntário, as informações relacionadas ao quadro clínico do idoso foram registada pela equipe de saúde do centro investigado.

Depois foi aplicado o VES-03, para identificar o idoso vulnerável ou não. O VES-03 propõe uma análise no perfil do idoso através de pontuações utilizando 4 dimensões: Idade em anos; Percepção da saúde; Limitação física e Incapacidade física. $O$ resultado da soma no VES-03 fica entre 0 à 10 pontos sendo que 0 à 2 pontos o idoso não é vulnerável e $\geq 3$ pontos o idoso é classificado como vulnerável. ${ }^{1,14}$

Em seguida, aplicou-se a FES-I-BR, a versão brasileira segue as mudanças feitas pela Rede Europeia de prevenção às quedas (PRoFaNE - Prevention of Falls Network Europe).

A FES-I-BR avalia o medo de cair durante a execução de 16 atividades sociais/externas, as pontuações variam de 1 a 4 pontos em cada questionamento, expressando desconforto sobre a possibilidade de sofrer uma queda: um ponto o indivíduo não está preocupado; dois está um pouco preocupado; três está muito preocupado e quatro pontos está extremamente preocupado em cair e a soma final alcança entre 16 a 64 pontos, em que 16 pontos correspondem à ausência de preocupação, 17-22 um pouco preocupado, 23-31 muito 
preocupado, 32-64 pontos extremamente preocupado em cair na realização das atividades. ${ }^{15,16}$

Os dados foram tabulados e organizados no programa Microsoft Excel for Windows, versão 10 e a análise estatística foi elaborada no software IBM - Statistical Package of Social Sciencs (SPSS 20.0) para Windows.

O cálculo do tamanho amostral do número de idosos avaliados em São Luís foi feito através do programa estatístico Power Analysis \& Sample Size (PASS 15 - 2017) e os seguintes parâmetros: Tamanho da população de idosos do grupo de convivência do CAISI 180 dos turnos matutino e vespertino, prevalência de quedas em idosos $72,3 \%,{ }^{17}$ nível de significância $(\alpha)$ de $5 \%$, poder do teste de $80 \%$, erro tolerável (erro padrão) de 7\%, com o tamanho mínimo da amostra de 114 mais 5\% de possíveis perdas, assim o número mínimo de idosos a serem avaliados segundo os cálculos estatísticos de 121 como ideal.

A amostragem foi do tipo não probabilístico. As variáveis categóricas foram distribuídas em frequência e porcentagem e as variáveis numéricas em média e desvio padrão. Para a comparação das 16 atividades da FES-IBR em função da classificação do VES-13 foi aplicado a análise no teste de MannWhitney $(U)$ e o teste $t$ de student independente nas variáveis numéricas.

Foi realizada regressão logística binária entre a variável dependente classificação da VES-13 grupo vulnerável e grupo não vulnerável com a variável independente classificação da FES-I-BR. Foi feita a correlação de Spearman entre as variáveis não paramétricas pontuação do VES-13 com pontuação da FESI-BR e entre a classificação do VES-13 com os resultados da FESI-BR e os dados sociodemográficos, por não apresentar uma distribuição de normalidade no teste de Kolmogorov-Smirnov.

A curva ROC (Receiver Operator Characteristic curve) foi utilizada para medir, especificar e descriminar vulneráveis de não vulneráveis, este protocolo foi feito por estudar a variação da sensibilidade e especificidade para diferentes valores de corte. Assim, a curva ROC foi utilizada em nosso estudo para analisar a capacidade do escore total da FES-I-BR, de discriminar pacientes com medo de cair entre vulneráveis de não vulneráveis.

A análise estatística foi realizada com um nível de confiança de $95 \%$, e foi considerado como significativo quando $p<0,05$.

\section{RESULTADOS}

Foram avaliados 123 idosos com uma média de idade de $69,98 \pm 6,87$ anos, com uma altura média de 1,51 $\pm 0,07$ e massa

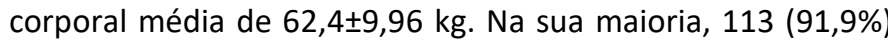
eram do sexo feminino, da faixa etária entre 60-74 anos 101 (82.1\%) e com a escolaridade $\geq 8$ anos estudado, 47 (38.2\%).

Em relação a raça/cor da pele a mais declarada foi a parda, 54 (43.9\%), a religião foi a católica, 100 (81.3\%), os estados civil mais declarados foram, casado, 34 (27.6\%) e o viúvo, 34 (27.6\%) e no histórico de quedas no último ano 59 (48\%) descreveram ter caído, o que pode ser observado na Tabela 1.

$\mathrm{Na}$ amostra total, a média do número de quedas no último ano foi de $(0,74 \pm 1,093)$, sendo $n=60(48,8 \%)$ caidores com média na pontuação da FES-I-BR de 26,63 $\pm 10,37$ e $n=63$ (51.2\%) não caidores com média na pontuação da FES-I-BR de $27,68 \pm 8,16$. Quanto ao medo de cair $n=120$ (97,6\%) apresentaram alguma preocupação em cair com pontuação média de $(27.11 \pm 9,26)$, desses $n=03(2.4 \%)$ estão classificados com ausência de preocupação, $n=38$ (30.9\%) um pouco preocupados, $n=46(37.4 \%)$ muito preocupados e $n=36$ (29.3\%) estão extremamente preocupados em cair.

Das 16 atividades abordadas na FES-I-BR os idosos apresentaram estar extremamente preocupados de cair ao realizar as seguintes atividades: andando sobre superfícies escorregadias (21.1\%), limpando casa (10.6\%) (Figura 1).

Tabela 1. Principais características e dados sociodemográficos da amostra estudada $(n=123)$

\begin{tabular}{|c|c|c|}
\hline Variáveis & $\mathbf{N}$ & $\%$ \\
\hline \multicolumn{3}{|l|}{ Faixa etária } \\
\hline $60-74$ anos & 101 & 82.1 \\
\hline $75-89$ anos & 21 & 17.1 \\
\hline 90 ou mais & 1 & .8 \\
\hline \multicolumn{3}{|l|}{ Sexo } \\
\hline Feminino & 113 & 91.9 \\
\hline Masculino & 10 & 8.1 \\
\hline \multicolumn{3}{|l|}{ Escolaridade } \\
\hline Nenhuma & 21 & 17.1 \\
\hline 1-3 anos de estudos & 21 & 17.1 \\
\hline 4-7 anos de estudos & 34 & 27.6 \\
\hline 8 anos ou mais de estudos & 47 & 38.2 \\
\hline \multicolumn{3}{|l|}{ Raça/cor } \\
\hline Negra & 40 & 32.5 \\
\hline Branca & 23 & 18.7 \\
\hline Parda & 54 & 43.9 \\
\hline Amarela & 1 & .8 \\
\hline Indígena & 4 & 3.3 \\
\hline Não declarada & 1 & .8 \\
\hline \multicolumn{3}{|l|}{ Religião } \\
\hline Católica & 100 & 81.3 \\
\hline Protestante & 19 & 15.4 \\
\hline Outra & 4 & 3.3 \\
\hline \multicolumn{3}{|l|}{ Estado Civil } \\
\hline Solteiro(a) & 30 & 24.4 \\
\hline Casado(a) & 34 & 27.6 \\
\hline Divorciado(a) & 25 & 20.3 \\
\hline Viúvo(a) & 34 & 27.6 \\
\hline \multicolumn{3}{|c|}{ Histórico de quedas no último ano } \\
\hline Sim & 59 & $48 \%$ \\
\hline Não & 64 & $52 \%$ \\
\hline
\end{tabular}

Quanto à vulnerabilidade, 41 (33,3\%) foram considerados

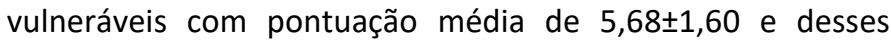
somente 17 (41,2\%) com históricos de quedas, e 82 (66.7\%) foram considerados como não vulneráveis com pontuação

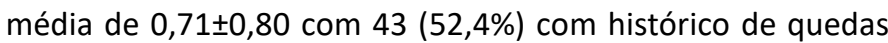
foi bem significativa a diferença entre as duas médias $(t=18,75$ $\mathrm{p}<0,0001$ ).

Quando realizada a comparação entre os idosos vulneráveis e não vulneráveis foi verificado que os vulneráveis apresentam mais medo de cair que não vulneráveis nas atividades limpando casa $(U=1293 ; p<0.05)$, indo as compras $(U=1214 ; p<0.01)$, subindo e descendo escadas $(U=1069.5 ; p<0.01)$, caminhando pela vizinhança $(U=1195 ; p<0.01)$, andando sobre superfícies escorregadias $(U=1202.5 ; p<0.01)$ e andando em lugares cheio de gente $(U=12335 ; p<0.01)$, para as demais atividades não foi observada diferença significativa entre os grupos (Tabela 2). 


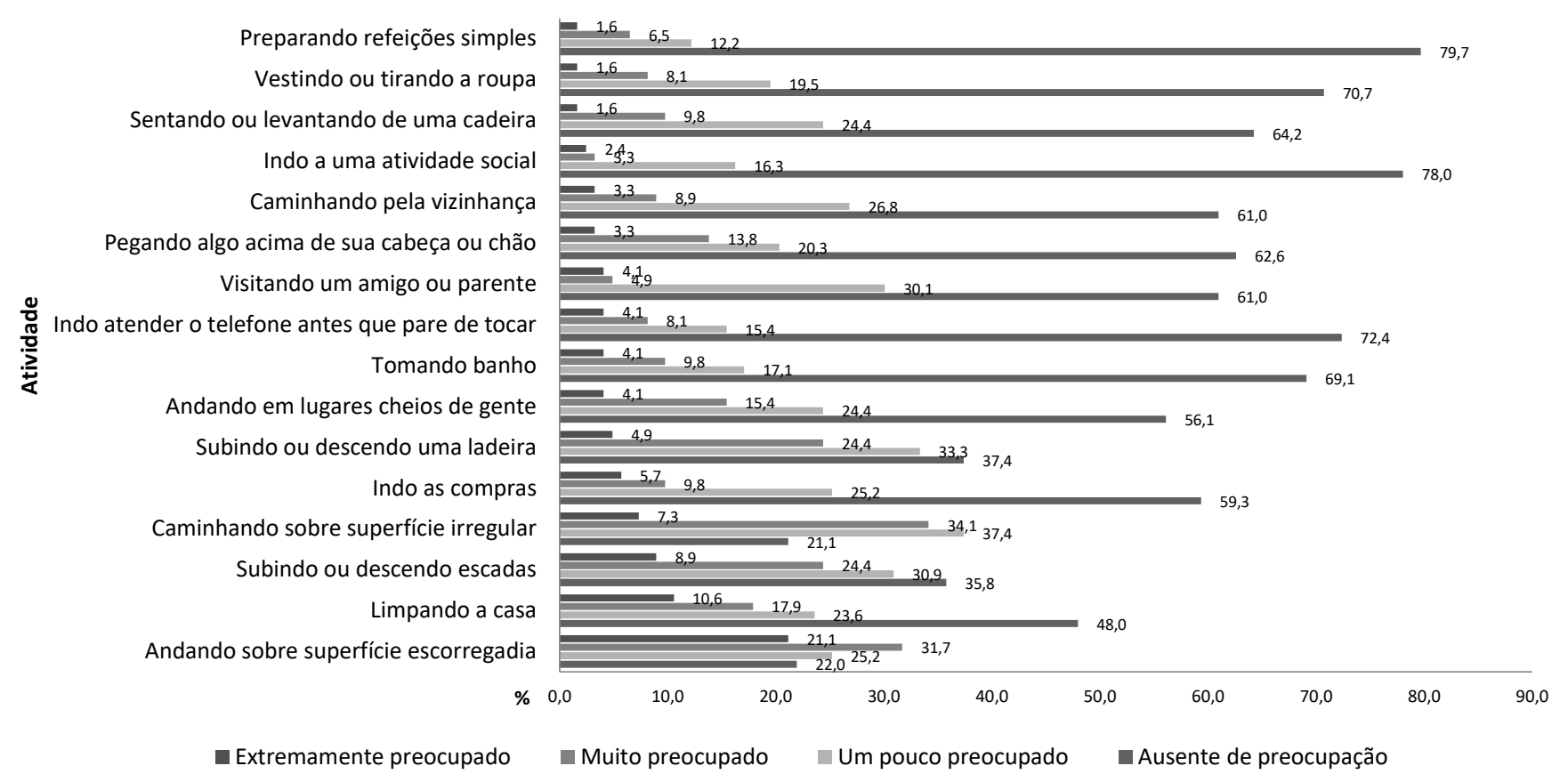

Figura 1. Frequência absoluta das 16 atividades avaliadas na FES-I-BR dos Idosos

Tabela 2. Distribuição das medianas das 16 atividades da FES-I-BR entre idosos não vulneráveis e vulneráveis

\begin{tabular}{|c|c|c|c|c|c|c|c|c|c|c|c|}
\hline \multirow{2}{*}{ Características } & \multicolumn{3}{|c|}{ Voluntários } & \multicolumn{3}{|c|}{ Não vulnerável } & \multicolumn{3}{|c|}{ Vulnerável } & \multirow{2}{*}{ u } & \multirow{2}{*}{ p-valor } \\
\hline & $25 \%$ & $50 \%$ & $75 \%$ & $25 \%$ & $50 \%$ & $75 \%$ & $25 \%$ & $50 \%$ & $75 \%$ & & \\
\hline Limpando a casa & 1,00 & 2,00 & 1,00 & 1,00 & 1,00 & 2,00 & 1,00 & 2,00 & 3,00 & 1293 & 0.026 \\
\hline Vestindo ou tirando a roupa & 1,00 & 1,00 & 2,00 & 1,00 & 1,00 & 1,00 & 1,00 & 1,00 & 2,00 & 1310.5 & 0.013 \\
\hline Preparando refeições simples & 1,00 & 1,00 & 1,00 & 1,00 & 1,00 & 1,00 & 1,00 & 1,00 & 2,00 & 1380 & 0.021 \\
\hline Tomando banho & 1,00 & 1,00 & 2,00 & 1,00 & 1,00 & 2,00 & 1,00 & 1,00 & 2,00 & 1437.5 & 0.109 \\
\hline Indo as compras & 1,00 & 1,00 & 2,00 & 1,00 & 1,00 & 2,00 & 1,00 & 2,00 & 3,00 & 1214 & 0.004 \\
\hline Sentando ou levantando de uma cadeira & 1,00 & 1,00 & 2,00 & 1,00 & 1,00 & 2,00 & 1,00 & 1,00 & 2,00 & 1372.5 & 0.05 \\
\hline Subindo ou descendo escadas & 1,00 & 2,00 & 3,00 & 1,00 & 2,00 & 2,00 & 2,00 & 3,00 & 3,00 & 1069.5 & 0.001 \\
\hline Caminhando pela vizinhança & 1,00 & 1,00 & 2,00 & 1,00 & 1,00 & 2,00 & 1,00 & 2,00 & 2,00 & 1195 & 0.003 \\
\hline Pegando algo acima de sua cabeça ou chão & 1,00 & 1,00 & 2,00 & 1,00 & 1,00 & 2,00 & 1,00 & 1,00 & 2,00 & 1620.5 & 0.707 \\
\hline Indo atender o telefone antes que pare de tocar & 1,00 & 1,00 & 2,00 & 1,00 & 1,00 & 1,00 & 1,00 & 1,00 & 2,00 & 1491 & 0.194 \\
\hline Andando sobre superfície escorregadia & 2,00 & 3,00 & 3,00 & 1,00 & 2,00 & 3,00 & 2,00 & 3,00 & 4,00 & 1202.5 & 0.008 \\
\hline Visitando um amigo ou parente & 1,00 & 1,00 & 2,00 & 1,00 & 1,00 & 2,00 & 1,00 & 1,00 & 2,00 & 1412.5 & 0.095 \\
\hline Andando em lugares cheios de gente & 1,00 & 1,00 & 2,00 & 1,00 & 1,00 & 2,00 & 1,00 & 2,00 & 3,00 & 1233.5 & 0.007 \\
\hline Caminhando sobre superfície irregular & 2,00 & 2,00 & 3,00 & 1,00 & 2,00 & 3,00 & 2,00 & 2,00 & 3,00 & 1402 & 0.114 \\
\hline Subindo ou descendo uma ladeira & 1,00 & 2,00 & 3,00 & 1,00 & 2,00 & 2,00 & 2,00 & 2,00 & 3,00 & 4630.5 & 0.010 \\
\hline Indo a uma atividade social & 1,00 & 1,00 & 1,00 & 1,00 & 1,00 & 1,00 & 1,00 & 1,00 & 2,00 & 4862.5 & 0.099 \\
\hline
\end{tabular}

U Mann-Whitney; $25 \%$ e $75 \%$ percentil; $50 \%$ Mediana; $p<0.05$ significante

Foi observada correlação positiva e significativa entre a pontuação do VES-13 com a pontuação da FES-I-BR $[\rho=0.287$; $p<0.01]$ demonstrando que quanto maior a pontuação do VES13 maior a pontuação da FES-I-BR, porém não foi observado resultados significativos entre a classificação da VES-13 e atividades individuais, bem como com os dados sociodemográficos (Tabela 3).

Também foi observado que o medo de cair foi um preditor significativo para a vulnerabilidade (OR=1,914; IC $95 \%=1,178$ 3,$111 ; p=0,009$ ) (Tabela 4). Em relação à curva ROC, foi encontrada uma área de $0,656(p<0,005)$ e observado que a FES-I-BR apresentou desempenho aceitável de avaliação entre vulneráveis e não vulneráveis.
Tabela 3. Correlação entre a classificação do VES-13 com dados sociodemográficos e os resultados da FES-IBR dos idosos do CAISI

\begin{tabular}{lcc}
\hline \multicolumn{1}{c}{ Variáveis } & p & Valor-p \\
\hline Pontuação do VES-13 x Pontuação da FES-I-BR & $0.287^{* *}$ & 0.001 \\
Classificação do VES-13 x Ausência de preocupação & -0.196 & 0.466 \\
Classificação do VES-13 x Um pouco preocupado & -0.84 & 0.757 \\
Classificação do VES-13 x Muito Preocupado & 0.197 & 0.465 \\
Classificação do VES-13 x Extremamente preocupado & 0.368 & 0.161 \\
Classificação do VES-13 x Escolaridade & -0.160 & 0.077 \\
Classificação do VES-13 x Raça/cor & -0.027 & 0.765 \\
Classificação do VES-13 x Religião & 0.011 & 0.900 \\
Classificação do VES-13 x Estado Civil & 0.061 & 0.506 \\
\hline
\end{tabular}

** Correlação significativa valor-p 0.01; $\rho$ Correlação de Spearman 
Tabela 4. A classificação do medo de cair entre idosos não vulneráveis e vulneráveis

\begin{tabular}{|c|c|c|c|c|c|c|}
\hline Variáveis & $\begin{array}{c}\text { FES-I-BR } \\
n=123(100 \%)\end{array}$ & $\begin{array}{c}\text { Não vulneráveis } \\
n=82(66.7 \%)\end{array}$ & $\begin{array}{c}\text { Vulneráveis } \\
n=41(33.3 \%)\end{array}$ & OR & IC 95\% OR & p-valor \\
\hline Ausência de preocupação & 3 & $02(66,7 \%)$ & $01(33,3 \%)$ & 1,914 & $1,178-3,111$ & 0,009 \\
\hline Um pouco preocupado & 38 & $31(81,6 \%)$ & $07(18,4 \%)$ & & & \\
\hline Muito preocupado & 46 & $31(67,4 \%)$ & $15(32,6 \%)$ & & & \\
\hline Extremamente preocupado & 36 & $18(50,0 \%)$ & $18(50,0 \%)$ & & & \\
\hline
\end{tabular}

O ponto de corte encontrado para discriminar vulneráveis de não vulneráveis foi de 28 ponto no escore total da FES-I-BR, com sensibilidade de $53,7 \%$ e especificidade $69,5 \%$ conforme (Figura 2) e a Tabelas 5 e 6.

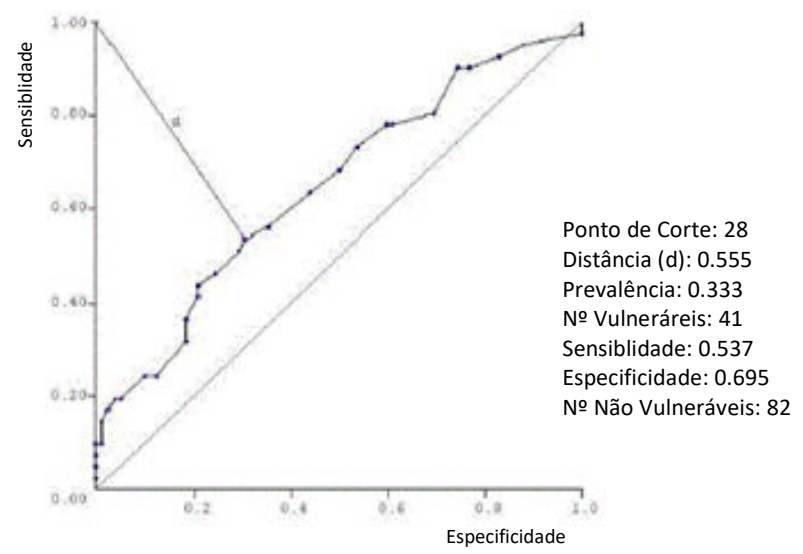

Figura 2. Curva ROC para avaliação da FES-I-BR com a classificação VES-132

\section{DISCUSSÃO}

A correlação entre o medo de cair com a vulnerabilidade em idosos foi observada, comprovando a hipótese deste artigo, assim quando maior a pontuação do medo de cair, maior a pontuação da vulnerabilidade, sugerindo que ao se deparar com um idoso que apresenta medo de cair deve ser diretamente levado em consideração a existência da vulnerabilidade, dessa forma, este trabalho pode auxiliar na avaliação mais rápida e eficaz das necessidades do idoso.

A maioria dos idosos apresentou algum medo de cair independente do histórico de quedas, corroborando com os resultados encontrados na pesquisa de Lopes, Costa e Castro ${ }^{9}$ onde a maioria dos voluntários também apresentou medo de cair em pelo menos uma das tarefas propostas pela FES-I-BR, em contrapartida, em outro estudo o medo de cair foi apresentado apenas no grupo de idosos com histórico de quedas. ${ }^{18}$ Embora seja comum encontrar o sentimento do medo por queda em idosos que já tenham caído, não é incomum encontrar o relato de medo de queda em idosos que nunca caíram, este sentimento de medo pode ser atribuído possivelmente pelas sequelas físicas e emocionais provocadas pelas fraturas relatadas por idosos com histórico de quedas como também propagado nas clínicas e hospitais. ${ }^{19}$

A proporção de idosos classificados como vulneráveis foi semelhante à encontrada em outros estudos ${ }^{20,21}$ e estes apresentaram mais medo de cair em relação aos não vulneráveis nas atividades limpando casa, indo as compras, subindo e descendo escadas, caminhando pela vizinhança,
Tabela 5. Área sob a curva, Variável (s) do resultado do teste: Score FES.I.BR

\begin{tabular}{ccccc}
\hline Área & Erro padrão & p-valor & \multicolumn{2}{c}{ Intervalo de confiança 95\% } \\
\hline \multirow{2}{*}{0,656} & 0,052 & 0,005 & Limite inferior & Limite Superior \\
& & & 0,554 & 0,758 \\
\hline
\end{tabular}

As variáveis do resultado do teste: Score da FES.I.BR tem pelo menos um empate entre o grupo de estados reais positivo e o grupo de estados reais negativo

Tabela 6. Precisão do Score da FES-I-BR para diferenciar participantes vulneráveis $(n=41)$ dos participantes não vulneráveis $(n=82)$

\begin{tabular}{|c|c|c|c|c|c|c|c|}
\hline 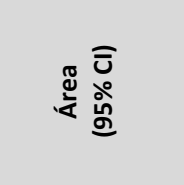 & 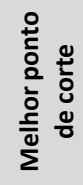 & 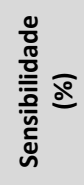 & 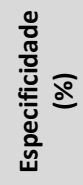 & $\begin{array}{l}\text { PPV } \\
\text { (\%) }\end{array}$ & $\begin{array}{l}\text { NPV } \\
\text { (\%) }\end{array}$ & PLR & NLR \\
\hline $\begin{array}{c}0.656 \\
(0.554-0.758)\end{array}$ & 28 & 53 & 69 & 46.78 & 75.04 & 1.76 & 0.67 \\
\hline
\end{tabular}

Cl: confidence interval; PPV: positive predictive value; NPV: negative predictive value; $P L R$ positive likelihood ratio; NLR: negative likelihood ratio

andando sobre superfícies escorregadias e andando em lugares cheio de gente, apontando que fatores de riscos relacionados ao ambiente e as atividades domésticas não apenas podem aumentar a prevalência de quedas ${ }^{22}$ como são identificadas pelos idosos como possíveis gatilhos causadores de queda.

De fato, neste estudo, a maioria dos idosos apresentaram algum medo de cair, porém os vulneráveis apresentaram significativamente mais medo de cair, como visto também em outros estudos, ${ }^{2,9}$ nas atividades que demandavam equilíbrio como andar em superfícies escorregadias, bem como força e coordenação muscular como limpar casa e subir ou descer escadas, demostrando que o idoso vulnerável, mesmo que empiricamente, percebe seu declínio funcional e com isso, o maior risco de sofrer uma queda. ${ }^{9,23}$ Entretanto, embora não tenham sido investigadas neste trabalho, também devem ser consideradas a influência do humor e mobilidade sobre a condição de vulnerabilidade.

Mesmo que a maioria dos idosos tenham sido considerados como não vulnerável, possivelmente devido a amostra desse estudo ser composta por idosos de um grupo de convivência, fisicamente ativos com suas funções cognitivas estimuladas de forma rotineira no centro de atenção a saúde do idoso, bem como o fato que a faixa etária estar abaixo dos 85 anos de idade que classifica o idoso como não vulnerável, ${ }^{24}$ ficou claro que os idosos vulneráveis apresentaram maior medo de cair, essas duas condições caminham juntas e podem refletir na necessidade de meios profiláticos e de cuidados. 
Além disso, a existência de uma correlação positiva e significativa entre o medo de cair com a vulnerabilidade ressalta que o medo de cair é uma característica do idoso vulnerável.

Portanto, o medo de cair foi constatado como um preditor significativo de vulnerabilidade uma vez que a FES-I-BR possibilitou a discriminação do medo de cair em idosos vulneráveis dos não vulneráveis, ou seja, ao ser identificado que o idoso possui medo de cair deve se atentar para a ocorrência da vulnerabilidade. possivelmente essas duas condições se correlacionam pela diminuição da autoconfiança e pelo declínio funcional. ${ }^{25}$

Como a vulnerabilidade no idoso está diferentemente relacionada com a ocorrência de dependência funcional, risco de institucionalização ou hospitalização, ${ }^{26}$ promovendo o aumento da probabilidade de óbito, este estudo se torna útil na avaliação e prevenção rápida da piora dos déficits relacionados a vulnerabilidade, principalmente os físicos relacionados ao equilíbrio e força, uma vez que ao detectar o medo de cair a vulnerabilidade já poderá ser predita.

Com os resultados apresentados, podemos observar as implicações clínicas relacionando a fragilidade provocadas pelo sentimento de medo com quedas nos idosos, como a diminuição de funções físicas caudas pela deambulação curta e lenta, posteriormente diminuição na mobilidade funcional que podem resultar em quedas, gerando com isso a diminuição na participação das atividades de vida diárias, isolamento social, depressão com risco de morte.

Essas complicações clínicas e sociais decorrentes ao isolamento social provocados pelo sentimento de medo ao realizar atividades rotineiras como limpar casa ou caminhar em superfícies escorregadias, aumenta o nível de suscetibilidades as doenças crônicas não transmissíveis, ao declínio funcional e posteriormente a quedas.

Oliveira et al. ${ }^{27}$ em seus achados, descrevem que a principal atividade nos acidentes domésticos é a deambulação e as principais barreiras ergonômicas caracterizadas como fatores extrínsecos para risco de quedas para idosos que vivem na comunidade são os pisos irregulares e escorregadios, além de atividades domésticas como descer e subir escadas, tomar banho e a ação de transferência de um local para outro.

Estudos futuros complementares são necessários para servirem como desfechos das causas provocados pelo medo de cair e para a identificação do idoso vulnerável em um contexto multidimensional para a garantia de sua saúde e qualidade de vida neste momento em vida, como a necessidade de pesquisa epidemiológica abrangente.

Como limitações deste estudo apontamos o fato de não ter sido inserido outro grupo de idosos usuários dos serviços do centro de referência investigado nesta pesquisa para comparamos entre os grupos a vulnerabilidade e o medo de cair e a possibilidade de viés nas informações referida relacionadas aos questionamentos utilizados sinalizadas na caderneta dos voluntários pelos profissionais de saúde do centro de referência ou autoreferidas quando estavam sem o preenchimento completo, porém, destacamos que este estudo apresenta amostra suficientemente grande para ser inferido para outras populações, além de ter utilizado ferramentas validadas.

\section{CONCLUSÃO}

O medo de cair está correlacionado com a vulnerabilidade e os idosos vulneráveis apresentaram mais medo de cair do que os não vulneráveis principalmente em atividades que demandem equilíbrio e força, adicionalmente o medo de cair é um preditor de vulnerabilidade o que torna a avaliação e a prevenção de déficits funcionais nos idosos mais rápida e eficaz.

\section{Agradecimentos}

Os autores agradecem pelo empenho dos colaboradores e pela equipe administrativa do CAISI, aos idosos que foram espontâneos a pesquisa, ao Programa do 20 ciclo acadêmico Mestrado em Meio Ambiente da Universidade Ceuma (UNICEUMA) e a Fundação de Amparo à Pesquisa e ao Desenvolvimento Científico e Tecnológico do Maranhão (FAPEMA) por proporcionarem realizações de pesquisas em São Luís, MA, Brasil.

\section{REFERÊNCIAS}

1. Lima CAB, Carvalho JL, Aquino RCA. Avaliação de vulnerabilidade do idoso através da adaptação transcultural do instrumento de identificação do idoso vulnerável VES-13. REER. 2017;3(1).

2. Cruz DT, Duque RO, Leite ICG. Prevalência do medo de cair em uma população de idosos da comunidade. Rev Bras Geriatr Gerontol. 2015;20(3):309-18. Doi: https://doi.org/10.1590/1981-22562017020.160176

3. Siqueira FV, Facchini LA, Piccin RX, Tomasi E, Thumé E, Silveira DS, et al. Prevalência de quedas em idosos e fatores associados. Rev Saúde Pública. 2007;41(5):749-56. Doi: https://doi.org/10.1590/S0034-89102007000500009

4. Santinha G, Marques $\mathrm{S}$. Ambiente construído, saúde pública e políticas públicas: uma discussão à luz de perceções e experiências de idosos institucionalizados. Saúde Soc. 2015;24(3):1047-60. Doi: https://doi.org/10.1590/S010412902015135520

5. Martins HDO, Bernado KM de A, Martins MS, Alfieri FM. Controle postural e o medo de cair em idosos fragilizados e o papel de um programa de prevenção de quedas. Acta Fisiatr. 2016;23(3):113-9.

6. Camargos FFO, Dias RC, Dias JMD, Freire MTF. Adaptação transcultural e avaliação das propriedades psicométricas da Falls Efficacy Scale - International em idosos brasileiros (FES-IBRASIL). Rev Bras Fisioter. 2010;14(3):237-43. Doi: https://doi.org/10.1590/S1413-35552010000300010

7. Franklin TA, Santos HCS, Santos Junior JA, Vilela ABA. Caracterização do atendimento de um serviço pré-hospitalar a idosos envolvidos em queda. Rev Fund Care Online. 2018; 10(1):62-67. Doi: http://dx.doi.org/10.789/21755361.2018.v10i1.62-67

8. Tinetti ME, Richman D, Powell L. Falls efficacy as a measure of fear of falling. J Gerontol. 1990;45(6):P239-43. Doi: http://dx.doi.org/10.1093/geronj/45.6.p239

9. Lopes K, Costa DF, Santos LF, Castro DP, Bastone AC. Prevalência do medo de cair em uma população de idosos da comunidade e sua correlação com mobilidade, equilíbrio dinâmico, risco e histórico de quedas. Rev Bras Fisioter. 2009;13(3):223-9. Doi: http://dx.doi.org/10.1590/S1413$\underline{35552009005000026}$ 
10. Tutiya KK, Barros BF, Santos FC. Queda em idosos: tradução, adaptação transcultural e validação do Teste de Rastreio "Q22p". RBM - Rev Bras Med. 2015;72(7):293-8.

11. Menezes C, Vilaça KHC, Menezes RL. Falls and quality of life of people with cataracts. Rev Bras Oftalmol. 2016;75(1):40-4. Doi: https://doi.org/10.5935/0034-7280.20160009

12. Reis MG, Carneiro C, Mayor MS, Pestana H. Quedas em idosos com perturbações mentais residentes em unidade de longa duração. Rev Port Enferm Saúde Mental. 2014;(Ed Esp 1):339.

13. Brasil. Ministério da Saúde. Caderneta de saúde da pessoa idosa: manual de preenchimento. Brasília (DF); Ministério da Saúde: 2008.

14. Sarges NA, Santos MIPO, Chaves EC. Evaluation of the safety of hospitalized older adults as for the risk of falls. Rev Bras Enferm. 2017;70(4):860-867.

Doi: https://doi.org/10.1590/0034-7167-2017-0098

15. Gonçalves AK, Hauser E, Martins VF, Possamai VD, Griebler EM, Blessmann EJ, et al. Postural balance program: Variables related to falls in elderly. J Phys Educ. 2017;28(1).1-10. Doi: https://doi.org/10.4025/iphyseduc.v28i1.2808

16. Hauser E, Sandreschi PF, Parizzotto D, Araújo CCR, Mazo GZ. Medo de cair e desempenho físico em idosos praticantes de atividade física. Rev Educ Fis/UEM.2015;26(4):593-600. Doi: https://doi.org/10.4025/reveducfis.v26i4.28442

17. Souza LHR, Brandão JCS, Fernandes AKC, Cardoso BLC. Queda em idosos e fatores de risco associados. Rev Bras Ciências da Saúde - USCS. 2017;15(54):55-60. Doi: https://doi.org/10.13037/ras.vol15n54.4804

18. Gonçalves AK, Griebler EM, Possamai VD, Costa RR, Martins VF. Idosos caidores e não caidores: programa de exercício multicomponente e prevalência de quedas. ConScientiae Saúde. 2017;16(2):187-93.

Doi: https://doi.org/10.5585/conssaude.v16n2.6987

19. Pinheiro SB, Cárdenas CJ, Akaishi L, Dutra MC, Martins WR. Avaliação do equilíbrio e do medo de quedas em homens e mulheres idosos antes e após a cirurgia de catarata senil. Rev Bras Geriatr Gerontol. 2016;19(3):521-32. Doi: https://doi.org/10.1590/1809-98232016019.150080
20. Wallace E, McDowell R, Bennett K, Fahey T, Smith SM. External validation of the Vulnerable Elder's Survey for predicting mortality and emergency admission in older communitydwelling people: a prospective cohort study. BMC Geriatr. 2017;17(1):69. Doi: https://doi.org/10.1186/s12877-0170460-1

21. Amancio TG, Oliveira MLC, Amancio VS. Fatores que interferem na condição de vulnerabilidade do idoso. Rev Bras Geriatr Gerontol. 2019;22(2)::e180159.

Doi: http://dx.doi.org/10.1590/1981-22562019022.180159

22. Morsch $P$, Myskiw M, Myskiw JC. A problematização da queda e a identificação dos fatores de risco na narrativa de idosos. Ciência \& Saúde Coletiva. 2016;21(11):3565-74. Doi: https://doi.org/10.1590/1413-812320152111.06782016

23. Galvão MH, Santos LIS, Barcelar JM, Marinho PEM. Avaliação da capacidade da Escala Internacional de Eficácia de Quedas em discriminar risco de quedas em pacientes com doença renal crônica submetidos à hemodiálise. Fisioter Pesq. 2013;20(2):151-7. Doi: https://doi.org/10.1590/S180929502013000200009

24. Wang J, Lin W, Chang LH. The linear relationship between the Vulnerable Elders Survey-13 score and mortality in an Asian population of community-dwelling older persons. Arch Gerontol Geriatr. 2018;74:32-38. Doi: https://doi.org/110.1016/j.archger.2017.09.005

25. Santos RKM, Maciel ACC, Britto HMJS, Lima JCC, Souza TO. Prevalência e fatores associados ao risco de quedas em idosos adscritos a uma Unidade Básica de Saúde do município de Natal, RN, Brasil. Rev Ciência \& Saúde Coletiva. 2015;20(12):3753-62. Doi: https://doi.org/10.1590/1413812320152012.00662015

26. Freitas FFQ, Soares SM. Índice de vulnerabilidade clínicofuncional e as dimensões da funcionalidade em idosos. Rev Rene. 2019;20:e39746. Doi: https://doi.org/110.15253/21756783.20192039746

27. Oliveira AS, Trevizan PF, Bestetti MLT, Melo RC. Fatores ambientais e risco de quedas em idosos: revisão sistemática. Rev Bras Geriatr Gerontol. 2014;17(3):637-45. Doi: https://doi.org/10.1590/1809-9823.2014.13087 